\title{
Orbital variations and impacts on observations from SNPP, NOAA 18-20, and AQUA sun-synchronous satellites
}

Xi Shao, Changyong Cao, Xiaoxiong Xiong, Tung-chang Liu, Bin Zhang, et al.

Xi Shao, Changyong Cao, Xiaoxiong Xiong, Tung-chang Liu, Bin Zhang, Sirish Uprety, "Orbital variations and impacts on observations from SNPP, NOAA 18-20, and AQUA sun-synchronous satellites," Proc. SPIE 10764, Earth Observing Systems XXIII, 107641 U (7 September 2018); doi: $10.1117 / 12.2323012$

EDIE Event: SPIE Optical Engineering + Applications, 2018, San Diego, California, United States 


\title{
Orbital Variations and Impacts on Observations from SNPP, NOAA 18-20, and AQUA Sun-synchronous Satellites
}

\author{
Xi Shao ${ }^{1}$, Changyong $\mathrm{Cao}^{2}$, Xiaoxiong Xiong ${ }^{3}$, Tung-chang Liu $^{1}$, Bin Zhang ${ }^{4}$, and Sirish Uprety ${ }^{1}$ \\ ${ }^{1}$ University of Maryland, College Park, MD, USA \\ ${ }^{2}$ NOAA/NESDIS/STAR, College Park, MD, USA \\ ${ }^{3}$ NASA Goddard Space Flight Center, Greenbelt, MD, USA \\ ${ }^{4}$ ERT Inc., Laurel, MD, USA
}

\begin{abstract}
The AQUA, SNPP, and NOAA 18-20 PM sun-synchronous satellites were designed with similar local time, local solar zenith angles, and overlapping temporal coverage. Although the satellites are expected to have fixed local equatorcrossing time, during the satellite lifetime, the equator-crossing times of these satellites drift. For NOAA 18-19, the drift in equator-crossing time is significant (few hours) and no correction has been done over the lifetime. For SNPP and AQUA, correction in the orbital inclination angle was periodically performed to maintain the equator-crossing time around the designed value. The impact of systematic drift of the local observation time during the satellite life cycle can be significant and should be accounted for when using multi-year time series of satellite products in long-term environmental studies. In this paper, the equator-crossing time drift of AQUA, SNPP, and NOAA 18-20, the correction of SNPP and AQUA equator-crossing time via orbital inclination angle change, and the consequent local solar zenith angle variation are evaluated. The impact of such drift on low-latitude mean brightness temperature trend derived from the similar $\sim 11 \mu \mathrm{m}$ thermal emissive channel of AQUA MODIS CH31, SNPP Visible Infrared Imaging Radiometer Suite (VIIRS) CH15 and NOAA 18-19 HIRS CH08 are analyzed. The drift in the mean brightness temperature measured by these sensors is combined as a function of local time and analyzed using diurnal cycle analysis. The mean brightness temperature drift for SNPP VIIRS is reconciled within the context of much larger temperature drift of NOAA 18-19.
\end{abstract}

Keywords: Orbital drift, Equator-crossing Time drift, Orbital inclination angle, Diurnal variation of mean brightness temperature, Brightness temperature drift

\section{INTRODUCTION}

The SNPP, NOAA 18-20, and AQUA satellites have been designed to be sun-synchronous with similar initial PM equator-crossing time. The AQUA mission was launched in May, 2002 and a part of the NASA-centered international Earth Observing System (EOS). NOAA 18-19 was launched in May, 2005 and February, 2009, respectively to support Earth environment monitoring and weather forecasting. SNPP and NOAA-20 serve as the next generation satellites to continue the legacy Earth observation by AUQA and NOAA18-19, and was launched in October, 2011 and November, 2017, respectively.

All of these LEO satellites carry VIS-NIR-IR imaging sensors and provide continuous global imagery monitoring of Earth environment in multiple spectral channels. For example, Moderate Resolution Imaging Spectroradiometer (MODIS) instrument onboard AUQA is a cross track scanning radiometer that collects data in 36 spectral bands with wavelengths from visible (VIS) to long wave infrared (LWIR) [1, 2]. High Resolution Infrared Radiation Sounder (HIRS) instrument onboard NOAA 18-19 collects multispectral data from one visible channel $(0.69 \mu \mathrm{m})$, seven shortwave channels $(3.7$ to $4.6 \mu \mathrm{m})$ and twelve longwave channels $(6.5$ to $15 \mu \mathrm{m})$ [3]. Visible Infrared Imaging Radiometer Suite (VIIRS) onboard SNPP and NOAA-20 supports operational environmental monitoring and numerical weather forecasting, with 22 imaging and radiometric bands covering wavelengths from 0.41 to 12.5 microns $[4,5]$. It

Earth Observing Systems XXIII, edited by James J. Butler, Xiaoxiong (Jack) Xiong, Xingfa Gu, Proc. of SPIE Vol. 10764, 107641U · (c) 2018 SPIE · CCC code: 0277-786X/18/\$18 · doi: 10.1117/12.2323012 
provides more than twenty sensor data records including clouds, sea surface temperature, ocean color, polar wind, vegetation fraction, aerosol, fire, snow and ice, vegetation, and other applications.

These LEO satellites were expected to have fixed local equator-crossing time. During the satellite lifetime, the equatorcrossing times of these satellites drift. For AQUA, SNPP and NOAA-20, correction in the orbital inclination angle was periodically performed to tightly maintain their equator-crossing time around the designed value. There are no active orbital correction for NOAA 18-19. Therefore, the equator-crossing time of NOAA 18-19 continues to drift and deviates as large as few hours from its initial value. The systematic drift of the local observation time during the satellite life can cause the satellite to progressively observe different phases of the solar illumination and diurnal cycle of the underlying surface and atmosphere. The modulated radiance measurement affect derived meteorological and climate products $[6,7]$. The impact of such drift can be significant and should be accounted for when using multi-year time series of satellite products in long-term environmental studies.

In this paper, long-term equator-crossing time drift and orbital inclination angle variation of AQUA, SNPP and NOAA18-20 are analyzed. The impacts of the orbital drift on long term local solar zenith angle change are evaluated. As an example of long term observational data affected by orbital drift, the mean brightness temperature (BT) trend derived from similar thermal emissive channel of AQUA MODIS, SNPP VIIRS, and NOAA 18-19 HIRS are analyzed. The drift in the mean BT is analyzed as a function of local time. The mean BT drift for 11 um channel of SNPP VIIRS is reconciled within the context of much larger temperature drift of NOAA 18-19.

\section{EQUATOR-CROSSING TIME VARIATION}

The change in the solar local time of observation can be closely tracked by the changes in the local time when the satellite crosses the equator $[6,7]$, i.e. equator-crossing time during its ascending orbit. To derive long term variation of equator-crossing time for a given LEO satellite, long term two-line-element (TLE) dataset of $\sim$ daily resolution has been collected from http://celestrak.com. Software based on SGP4 orbital prediction code has been developed to predict the satellite equator-crossing time using the continuous long term TLE data. Figure 1 shows the local equator-crossing time drift of AQUA, SNPP and NOAA18-20 as derived from the TLE data of each satellite. Due to lack of orbital correction, the equator-crossing time of NOAA 18-19 is the most significant. Since its launch, the equator-crossing time of NOAA 18 drifts from initial 14:00 PM to 13:30 PM and then to $19: 50 \mathrm{PM}$, i.e. terminator orbit. The equator-crossing time of NOAA 19 varies from to initial 14:00 PM to 13:30 PM and then to current 16:00 PM over nine years. On the other hand, the equator-crossing time drift for SNPP accumulates to $\sim 10 \mathrm{~min}$ in late 2014 when major orbital adjustments were applied. The follow-up adjustments were applied in $\sim$ yearly interval to maintain the equator-crossing time of SNPP around 13:25 PM. Before 2012, the equator crossing time of AQUA is between 13:31:48 and 13:40:48 PM. Since 2012, the equator crossing time of AQUA has been tightly maintained between 13:35:00 and 13:36:30 PM. Since its launch in November, 2017, the equator-crossing time of NOAA-20 is within 1 minute around 13:25 PM.

The satellite TLE data can also be analyzed to extract the variation of orbital inclination angle. Time history of orbital inclination angle can reveal the periodical orbtial adjusments to maitian the eqautor crossing time of AQUA and SNPP. Figure 2 shows the zoom-in view of equator-crossing time and orbital inclination angle variation of SNPP since 2012. Adjustment in SNPP orbital inclination angle helps maintain its equator-crossing time at designed value. Six adjustments of SNPP orbital inclination angle and the corresponding changes in equator-crossing time can be identified in Figure 2. The equator-crossing time of SNPP drifts from initial 13:25 PM to as large as 13:33 PM in late 2014 and the corresponding orbital inclination angle changes from 98.675 degree to 98.79 degree. Four consecutive orbital adjustments were applied in late 2014 to bring the orbital inclination angle to 98.68 degree and the equator-crossing time drifted afterwards toward 13:25 PM. The follow-up orbital adjustment was applied in yearly interval to maintain its equator-crossing time at the targeted value, which can be identified from the yearly discrete change in the orbital inclination angle in Figure 2.

Figure 3 shows the orbital inclination angle variation of AQUA and NOAA 19 since 2012. The orbital adjustment of AUQA occurs yearly which helps maintain a tighter equator-crossing time drift than SNPP. For AQUA, the orbital inclination angle was maintained between 98.19 and 98.23 degree and when the inclination angle approached 98.23 degree, four to five consecutive orbital adjustments were performed to maintain the equator-crossing time around the designed value. 
There is no orbital correction for NOAA 19. Over six years since 2012, NOAA 19 equator-crossing time drifts about 2.5 hours as the orbital inclination angle continuously increases from 98.85 degree to 99.09 degree as shown in Figure 3.

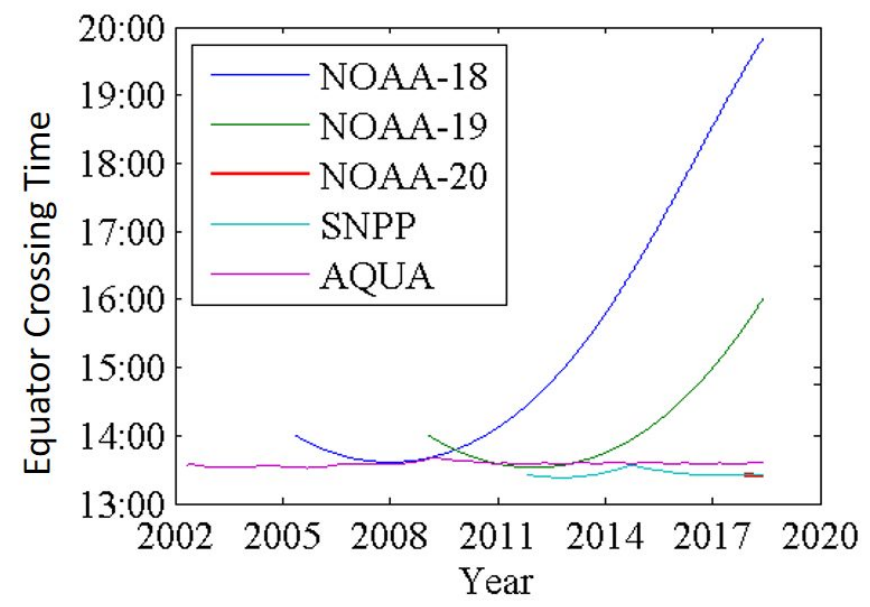

Figure 1: Equator-crossing time variation for the ascending orbit of AQUA, SNPP and NOAA 18-20.

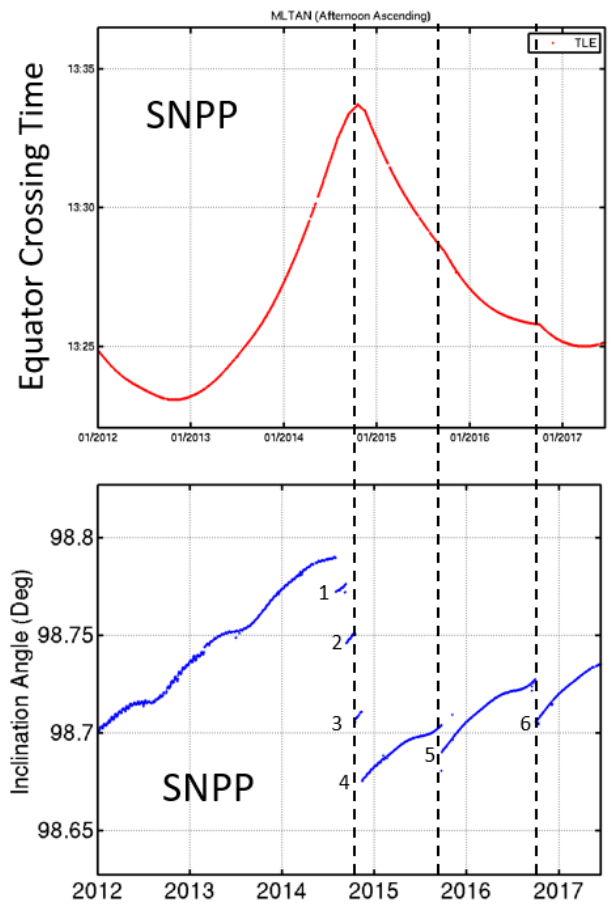

Figure 2: Top: Equator-crossing time variation of SNPP. The equator-crossing time variation range is $\sim 10$ min for SNPP. Bottom: Orbital inclination angle variation for SNPP. The inclination angle variation is as large as $\sim 1$ degree for SNPP. The discrete adjustments in orbital inclination angle are labeled as 1-6. 


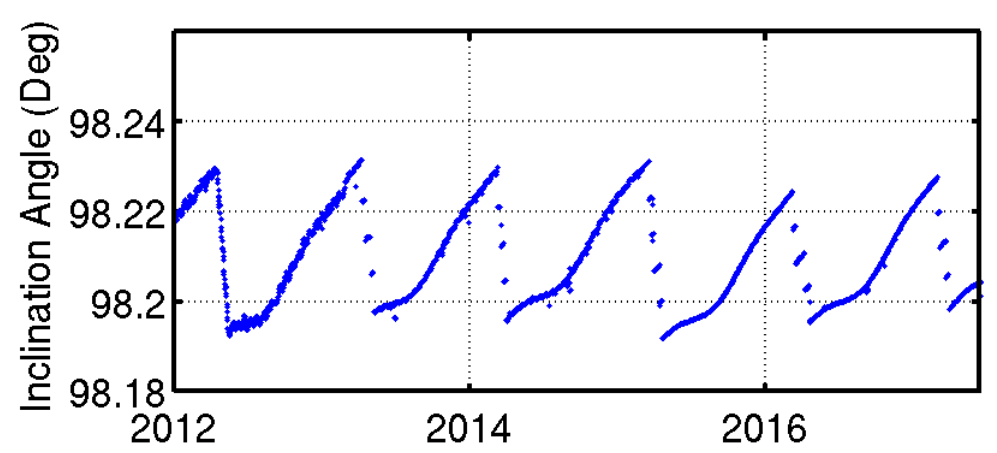

(a) Orbital Inclination Angle Variation of AQUA

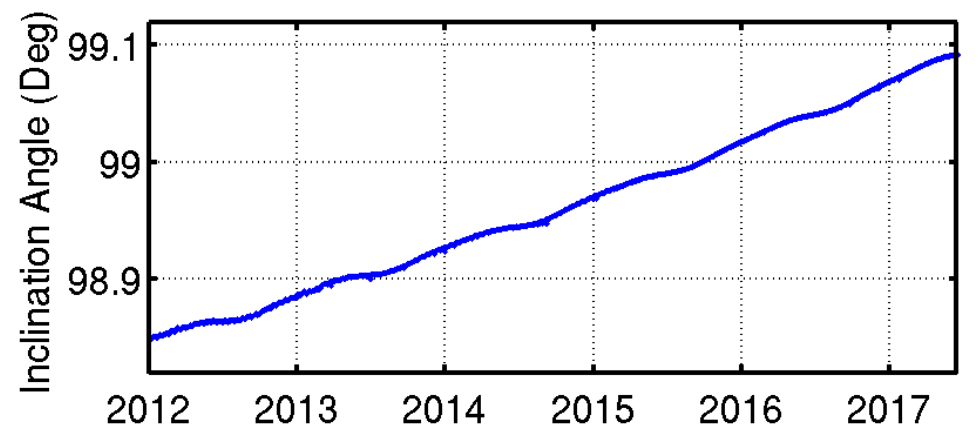

(b) Orbital Inclination Angle Variation of NOAA-19

Figure 3: Orbital inclination angle variation of AQUA (top) and NOAA-19 (bottom).

\section{LOCAL SOLAR ZENITH ANGLE VARIATION}

A direct consequence of the LEO satellite equator-crossing time variation can be reflected in the change of the local solar zenith angle when the satellite makes measurements. To evaluate the variation in local zenith angle, orbital prediction from TLE data was further analyzed to calculate the mean solar zenith angle at the satellite nadir averaged in different latitude regions. The local solar zenith angle has large annual variation due to the annual variation in the solar declination angle, which can mask the embedded small solar zenith angle change due to the orbital drift. Therefore, a filter has been applied to remove the annual oscillation in the solar zenith angle variation. The resulting filtered local solar zenith angle variations for three LEO satellites in six latitude regions are shown in Figure 4. The overall variation range of the mean solar zenith angle is about 10 degree when the selected latitude region varies between -30 to 30 degree in latitude. The latitude region in the southern hemisphere has higher solar zenith angle, which is due to the inclination of the satellite orbital plane.

Since the equator-crossing time of AQUA was tightly controlled, the solar zenith angle maintains almost unchanged as shown in Figure 4b. The equator-crossing time drift of SNPP was the largest in late 2014. The variation of the solar zenith angle peaked with a maximum of $\sim 2.5$ degree in late 2014 which corresponds to the equator-crossing time drift of $\sim 10$ min. The mean local solar zenith angle of NOAA 19 continues to increase due to the uncontrolled orbital drift. With $\sim 2.5$ hour drift in the equator-crossing time of NOAA 19 since 2012, the deviation in the solar zenith angle from its initial value is about 20 degree. 


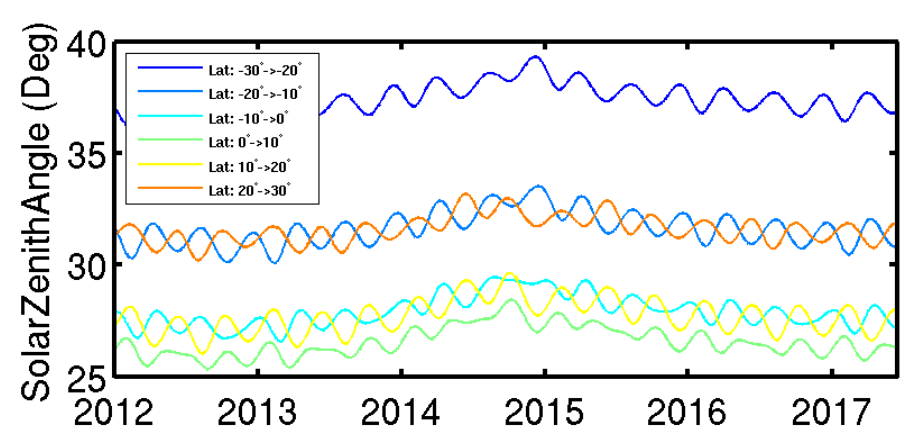

(a) SNPP

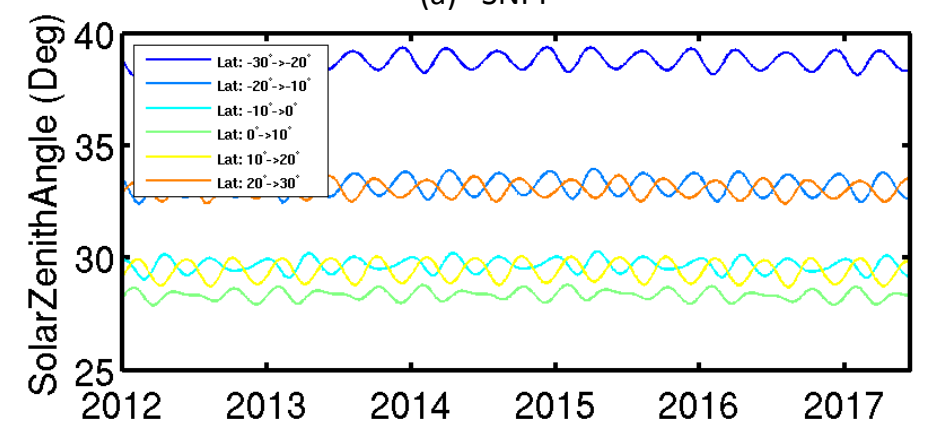

(b) AQUA

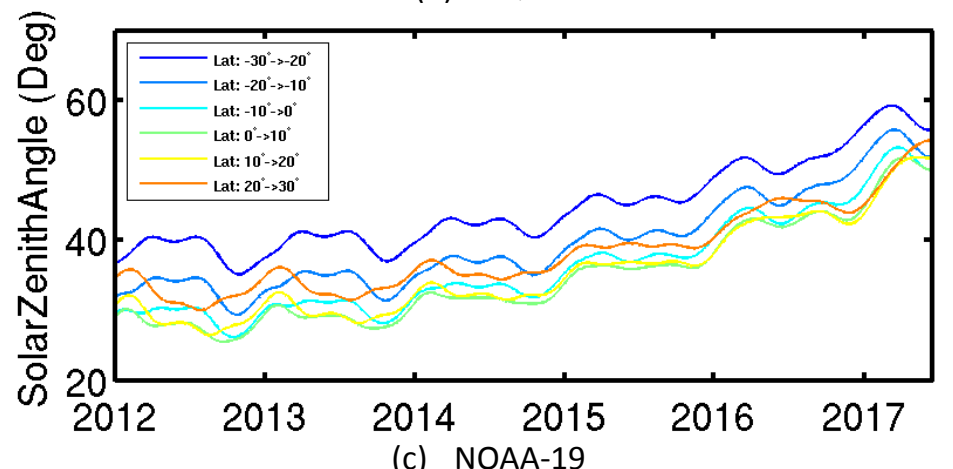

Figure 4: Mean solar zenith angle variation at nadir of SNPP, AQUA and NOAA-19 in different latitude regions. Annual oscillation in the data has been filtered out as much as possible with the annual oscillation removal filter.

\section{LOW-LATITUDE MEAN BRIGHTNESS TEMPERATURE DRIFT}

\subsection{Data used in Mean Brightness Temperature Analysis}

The local solar zenith angle variation due to the orbital drift of sun-synchronous satellite can cause the satellite to progressively observe different phases of the diurnal cycle of the underlying surface and atmosphere. Such systematic drift of the local observation time during satellite life cycle can have significant impact. In this study, observational data of the similar $\sim 11$ um thermal emissive channel from multiple satellites were analyzed to study the impact of the equator-crossing time drift on low-latitude mean BT trend. This $\sim 11 \mu \mathrm{m}$ channel is primarily used for surface temperature retrieval. Table 1 listed the channel number and central wave number of the chosen channel for AQUA MODIS, SNPP VIIRS, and NOAA 18-19 HIRS. The SNPP VIIRS CH15 data used in this analysis have been consistently reprocessed from its launch to September 30, 2016. The AQUA MODIS CH31 data used in this analysis is collected from the latest Version 6.1 dataset. 
Since these four instruments have overlapping temporal coverage since 2012, the observational data after 2012 were collected for the chosen channel of these four instruments. During each scan of the imaging sensor, data centered around satellite nadir spanning $\sim 85 \mathrm{~km}$ in ground distance on either side of the nadir were collected. Observational UT time, scan-averaged longitude/latitude and scan-averaged BT were recorded for each scan. The UT time and mean longitude were later used to calculate local time and the mean latitude was used in determining latitude region. Scan-averaged BT data were accumulated over calendar month and further averaged according to the selected latitude region. The resulting monthly/latitude region-averaged BT and the corresponding mean local time data were used in both time series and diurnal cycle analysis.

\begin{tabular}{|l|c|l|c|c|}
\hline & AQUA MODIS & SNPP VIIRS & $\begin{array}{l}\text { NOAA-18 } \\
\text { HIRS }\end{array}$ & $\begin{array}{l}\text { NOAA-19 } \\
\text { HIRS }\end{array}$ \\
\hline Channel \# & 31 & 15 & 8 & 8 \\
\hline $\begin{array}{l}\text { Central Wave } \\
\text { Number }\left(\mathrm{cm}^{-1}\right)\end{array}$ & 906.62 & 929.97 & 900.46 & 899.46 \\
\hline
\end{tabular}

Table 1: Channel number and central wave number of the corresponding $\sim 11$ um channel selected for the four imaging instruments of interest.

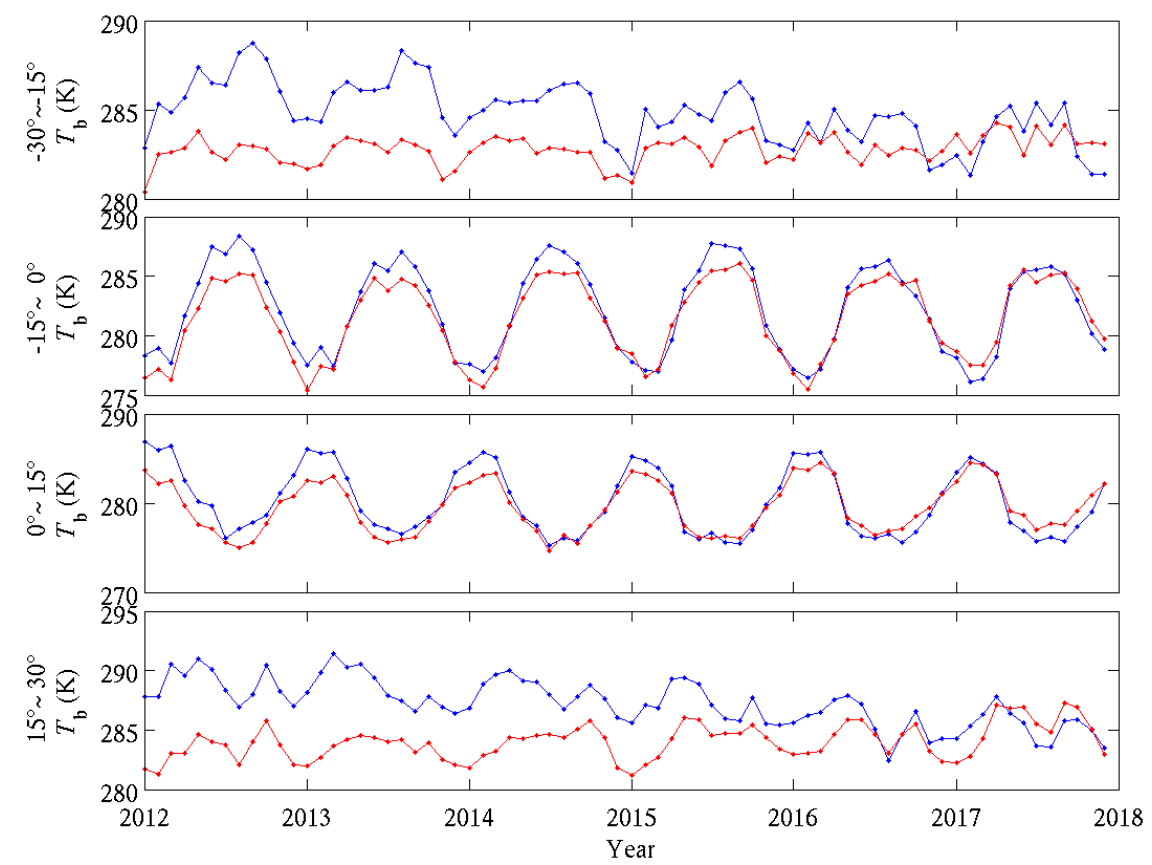

Figure 5: Time history of monthly mean brightness temperature in different latitude regions as measured by NOAA-18 HIRS CH08. Blue and red line in each panel show the variation of the mean BT collected during satellite ascending and descending orbit, respectively.

Figure 5 shows example of monthly mean CH08 BT time history in different latitude regions as measured by NOAA-18 HIRS. There is strong annual cycle oscillation in the BT of low latitude regions (-15 to 0 and 0 to 15 degree in latitude) in comparison with the BT of higher latitude regions. The annual oscillation has opposite phase in northern and southern hemisphere due to the annual variation of solar declination angle. Since the satellite overpasses the same latitude region during both ascending and descending orbits which are $\sim 12$-hour apart in local time, the time variation of mean BT sampled during ascending and descending orbit is shown in Figure 5 as blue and red curve, respectively. Due to the orbital drift of NOAA18, its equator-crossing time of ascending orbit drifted from its initial location at 14:00 PM to 19:30 PM in late 2017 when NOAA 18 satellite became terminator crossing satellite. Such drift can be clearly seen from the variation of mean BT in Figure 5. The BT sampled during ascending orbit continued to decrease while the BT sampled during descending orbit continued to increase. When NOAA 18 approaches the terminator orbit, there is a cross-over between the BT sampled during ascending and descending orbits. In southern hemisphere, the BT sampled at 
local time around 7:30 AM during descending orbit became higher than the BT sampled around 19:30 PM during ascending orbit.

\subsection{Low-Latitude Mean Brightness Temperature Variation}

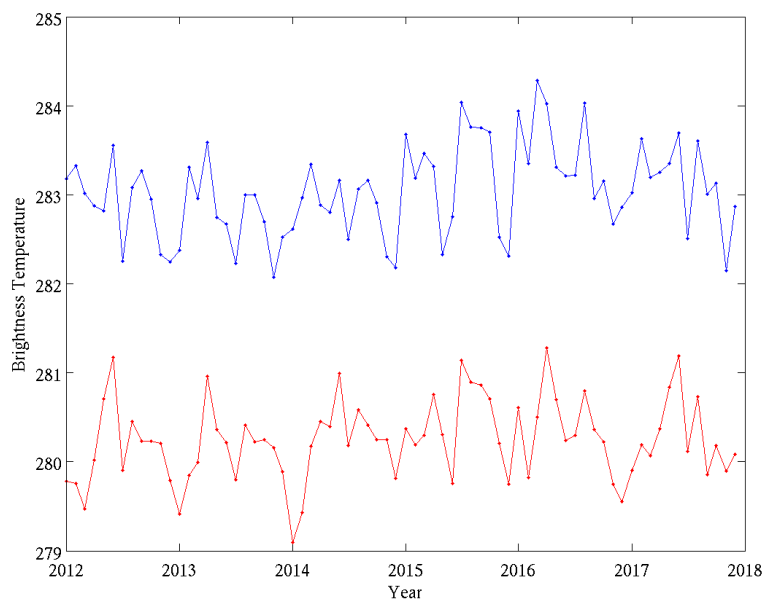

(a) Aqua MODIS

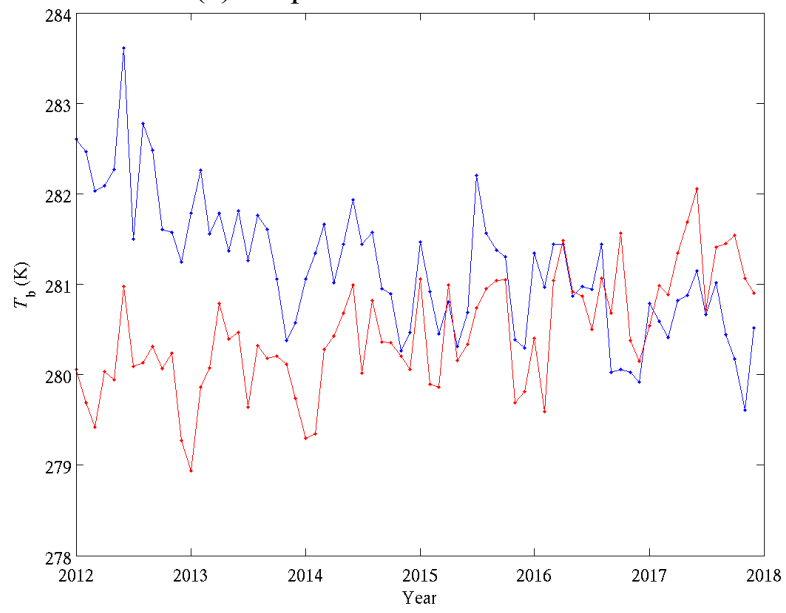

(c) NOAA-18

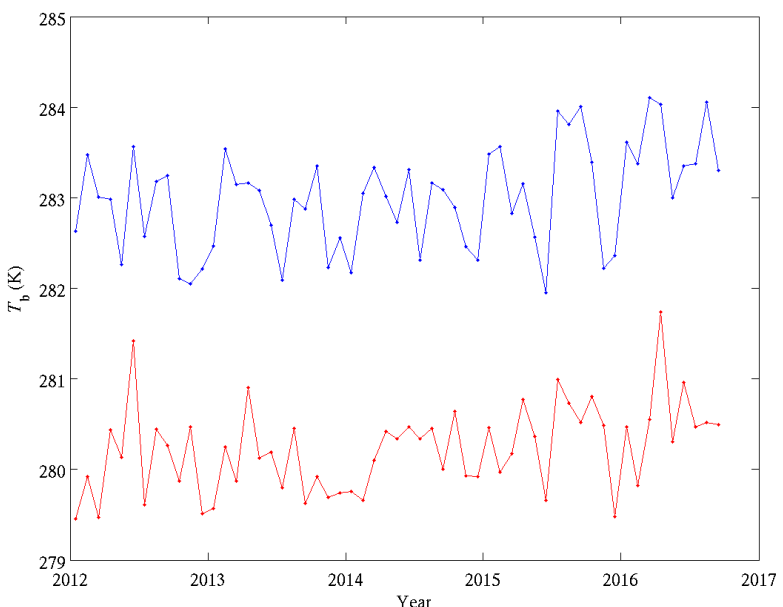

(b) SNPP VIIRS

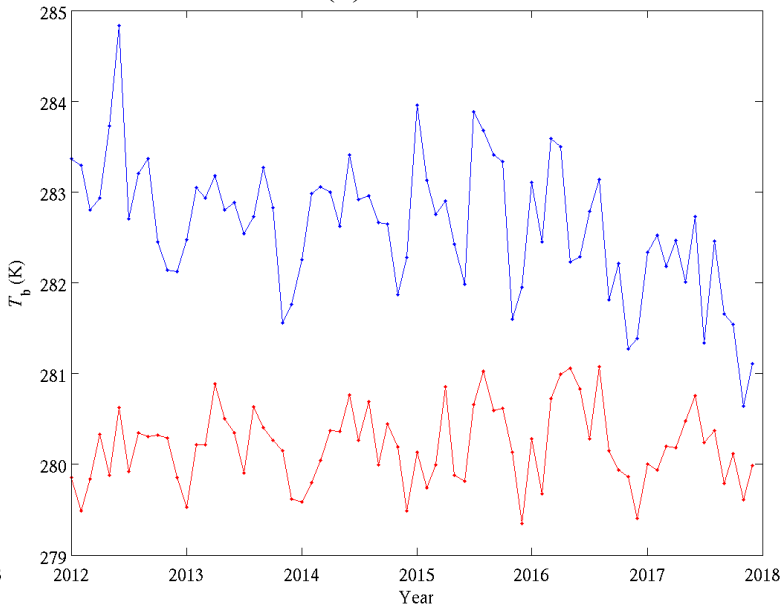

(d) NOAA-19

Figure 6: Time history of low-latitude mean brightness temperature derived from measurements by four instruments. The low-latitude mean BT is calculated by averaging BT over latitude region within - 15 to 15 degree in latitude. In all panels, blue and red line shows the time history of the mean TB collected during satellite ascending and descending orbit, respectively.

By averaging the $\mathrm{BT}$ in low latitude region consisting of both northern and southern hemisphere, the annual cycle oscillation in BT can be canceled out to certain extent. Figure 6 shows the time series of mean BT in the low latitude region within latitudes from -15 to 15 degree as derived from measurements by the four instruments. The amplitude of annual oscillation in the low latitude mean BT data of NOAA 18 can be as large as $8 \mathrm{~K}$ over one side of hemisphere as shown in Figure 5. After combining the data from both hemispheres, the remnant fluctuation in the low latitude mean BT is less than $2 \mathrm{~K}$. The long term trend due to the orbital drift can be clearly identified in the low latitude mean BT of NOAA 18 and 19. Both show continuous decreasing and increasing trend in the data collected during ascending and descending orbit, respectively. The cross over between the ascending and descending low latitude mean BT of NOAA 18 can also be identified when it drifts to the terminator orbit. The remnant fluctuation in the low latitude mean BT can be attributed to the slightly different overpassing local time originated from the inclined orbital plane and difference in surface feature between low latitude regions in northern and southern hemisphere. Such fluctuation is relatively large 
and masks the subtle long term trend embedded in the SNPP VIIRS data that is due to a 10-minute drift in equator crossing time.

\subsection{Diurnal Variation of Low Latitude Mean Brightness Temperature}

The low latitude mean BT derived from the measurements of the four instruments can be combined and analyzed using diurnal cycle analysis. To reconcile the low latitude mean BT as a function of local time due to orbtial drift, the monthly mean BT data from AQUA MODIS, SNPP VIIRS and NOAA 18-19 HIRS were combined relative to the mean local time as shown in Figure 7.

Following [8,9], the diurnal cycle of BT variation was fitted with a second order Fourier series (Eq. 1).

$$
T_{b}(t)=a_{0}+a_{1} \cos \frac{\pi\left(t-t_{1}\right)}{12}+a_{2} \cos \frac{2 \pi\left(t-t_{2}\right)}{12}
$$

where $a_{0}$ is the mean level of BT; $a_{1}$ and $a_{2}$ are the magnetitude of the 24- and 12- hour oscillation with peak at local time $=t_{1}$ and $t_{2}$, respectively.

The black line shown in Figure 7 is the fitted diurnal curve with Eq. (1) using the cobined low latitude mean BT data from four insturments. Since the NOAA 18 and 19 instruments have larger local time drift, the fitted curve is dominated by the data from HIRS of these two satellites. The fitted diurnal curve reconciles well the combined low latitude mean BT data of ascending and descending orbits. Furthermore, the mean BT drift for SNPP VIIRS can be estimated within the context of much larger temperature drift of NOAA 18-19. With the fitted function of diurnal variation and given the equator crossing time drift of 10 minutes, the drift in low latitude mean BT for SNPP VIIRS is estimated to be $0.084 \mathrm{~K}$ and $0.028 \mathrm{~K}$ for the ascending and descending orbit, respectively.

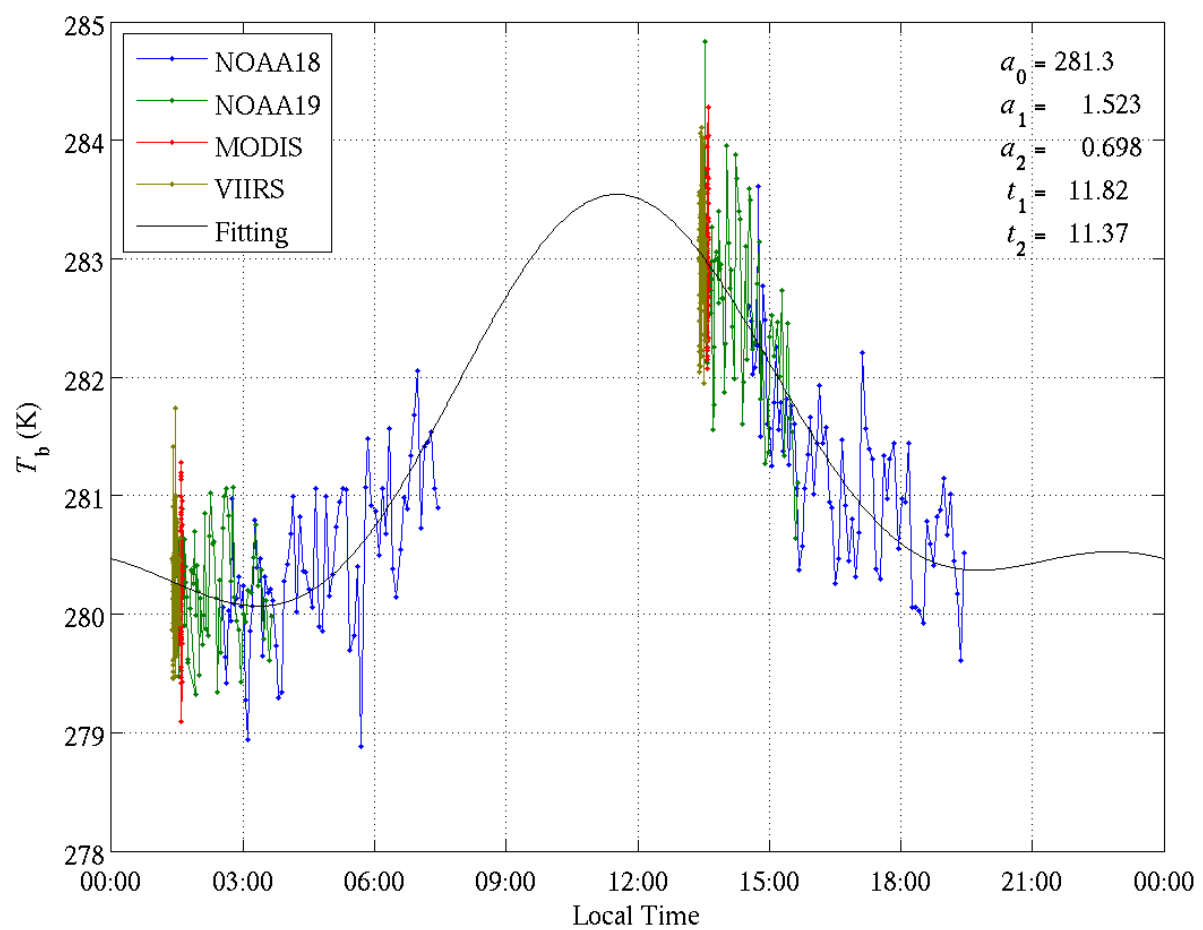

Figure 7: Low latitude mean BT of $\sim 11$ um channel of AQUA MODIS, SNPP VIIRS and NOAA18-19 HIRS collected over six years from 2012 to 2017 vs. mean local time. Fitted diurnal variation of low latitude BT using Eq. (1) is shown as black line. Fitting coefficients are listed in the figure. 


\section{SUMMARY}

In this paper, long-term equator-crossing time variation of AQUA, SNPP and NOAA18-20 due to orbital drift are compared. The discrete orbital inclination angle adjustments to tightly maintain equator-crossing time for AQUA and SNPP were identified. In comparison, there were no orbital inclination angle adjustments for NOAA 18-19. Their orbital inclination angle continues to increase together with the equator-crossing time drifting toward terminator orbit. The impacts of orbital drift on the local solar zenith angle of satellite measurements were also evaluated. As an example of long term observational data affected by orbital drift, low-latitude mean brightness temperature trend derived from similar $\sim 11 \mu \mathrm{m}$ thermal emissive channel of AQUA MODIS, SNPP VIIRS and NOAA 18-19 HIRS were analyzed. The long term low latitude mean BT of NOAA 18 and 19 showed continuous decreasing and increasing trend for the data collected during ascending and descending orbit, respectively. There is a cross over between the ascending and descending low latitude mean BT of NOAA 18 when it drifts to the terminator orbit. The drift in the mean BT measured by four instruments is combined as a function of local time. The mean BT drift for SNPP VIIRS was estimated within the context of much larger temperature drift of NOAA 18-19. With the equator crossing time drift being 10 minutes, the drift in low latitude mean BT for SNPP VIIRS is estimated to be $0.084 \mathrm{~K}$ and $0.028 \mathrm{~K}$ for the ascending and descending orbit, respectively. Further analysis will be performed to study the impacts of orbital drift on the long term BT retrieval at selected sites such as desert with cloud masking and global mean BT.

\section{ACKNOWLEDGEMENT}

The manuscript contents are solely the opinions of the authors and do not constitute a statement of policy, decision, or position on behalf of NOAA or the U.S. government.

\section{REFERENCES}

[1] Xiong, X., Wu, A., and Cao, C., "On orbit calibration and inter comparison of Terra and Aqua MODIS surface temperature spectral bands," International Journal of Remote Sensing 29(17-18), 5347-5359 (2008).

[2] Xiong, X., Wenny, B. N., Wu, Aisheng, Barnes, W. L., and Salomonson, V. V., "Aqua MODIS Thermal Emissive Band On-Orbit Calibration, Characterization, and Performance," IEEE Transactions on Geoscience and Remote Sensing 47(3), 803-814 (2009).

[3] Cao, C., Xu, H., Sullivan, J., McMillin, L., Ciren, P., and Hou, Y., "Intersatellite radiance biases for the High Resolution Infrared Radiation Sounders (HIRS) onboard NOAA-15, -16, and -17 from simultaneous nadir observations," Journal of Atmospheric and Oceanic Technology 22(4), 381-395 (2005).

[4] Cao, C., Xiong, X., Blonski, S., Liu, Q., Uprety, S., Shao, X., Bai, Y., and F. Weng, "Suomi NPP VIIRS Sensor Data Record Verification, Validation and Long Term Performance Monitoring," Journal of Geophysical ResearchAtmosphere 118(20), 11664-11678 (2013).

[5] Cao, C., DeLuccia, F., Xiong, X., Wolfe, R., and Weng, F., "Early On-orbit Performance of the Visible Infrared Imaging Radiometer Suite (VIIRS) onboard the Suomi National Polar-orbiting Partnership (S-NPP) Satellite," IEEE Trans. Geosci. and Remote Sens. 52(2), 1142-156 (2014).

[6] Ignatov, A., Laszlo, I., Harrod, E. D., Kidwell, K. B., and Goodrum, G. P., "Equator crossing times for NOAA, ERS and EOS sun-synchronous satellites," International Journal of Remote Sensing 25(23), 5255-5266 (2004).

[7] Nagol, J., Vermote, E., and Prince, S., "Quantification of Impact of Orbital Drift on Inter-Annual Trends in AVHRR NDVI Data," Remote Sens. 6, 6680-6687 (2014).

[8] Lindfors, A. V., MacKenzie, I. A., Tett, S. F. B., and Shi, L., "Climatological diurnal cycles in clear-sky brightness temperatures from the high-resolution infrared radiation sounder (HIRS)," J. Atmos. Oceanic Technol. (28), 11991205 (2011).

[9] MacKenzie, I. A., Tett, S. F. B., and Lindfors, A. V., "Climate model-simulated diurnal cycles in HIRS clear-sky brightness temperatures,” J. Climate (25), 5845-5863 (2012). 